\section{Kidney metabolite induces FGF23 rise}

In patients with chronic kidney disease, impaired phosphate clearance leads to high serum levels of the phosphaturic hormone fibroblast growth factor 23 (FGF23). Elevated FGF23 is also associated with an increased risk of acute kidney injury (AKI) and worse outcomes in AKI survivors. A new study by Eugene Rhee and colleagues suggests that kidney-derived glycerol 3-phosphate (G3P) induces FGF23 production in bone.

The researchers measured proteins and metabolites in aortic and renal venous samples from 17 individuals and found that G3P was the only molecule that correlated significantly with arterial levels of FGF23; this correlation was stronger than that between arterial phosphate and FGF23 levels. Renal venous $\alpha$-Klotho did not correlate with either renal venous G3P or arterial FGF23.

In mice, a single injection of $\mathrm{G} 3 \mathrm{P}$ increased plasma FGF23 levels, which was associated with a rise in urinary phosphate and a decrease in plasma calcitriol but had no effect on parathyroid hormone levels. The researchers then investigated a potential role for lysophosphatidic acid (LPA), which is synthesized by $\mathrm{G} 3 \mathrm{P}$ acyltransferase (GPAT), downstream of G3P. Injection of G3P increased LPA levels in bone but not in plasma and this effect was blocked by a GPAT inhibitor. Plasma FGF23 levels also increased in response to LPA injection, but only in mice with intact Lpar1, which encodes the most highly expressed LPA receptor in bone.

Using bilateral ischaemia-reperfusion injury (IRI) as a mouse model of AKI, the researchers showed that IRI significantly increased kidney and plasma G3P levels compared with controls; this rise was LPAR1-dependent and was blunted in the presence of a GPAT inhibitor. Following cardiac surgery, plasma G3P levels were also significantly higher in patients who subsequently developed AKI than in those who did not.

"G3P released by the kidney stimulates bone production of FGF23 via local conversion of G3P to LPA and binding to LPAR1 - these results may highlight a new kidney endocrine function," concludes Rhee. "We plan to investigate whether inhibition of this G3P-FGF23 axis is beneficial in AKI."

Monica Wang

ORIGINAL ARTICLE Simic, P. et al. Glycerol-3-phosphate is an FGF23 regulator derived from the injured kidney. J. Clin. Invest. https://doi.org/10.1172/JCl131190 (2020)

\title{
GLOMERULONEPHRITIS
}

\section{Bacterial infection linked to anti-factor B antibodies}

Acute postinfectious glomerulonephritis (APIGN) and C3 glomerulopathy (C3G) are both characterized by low serum $\mathrm{C} 3$ levels at diagnosis. However, this decrease is transient in patients with APIGN, whereas C3 levels are persistently low in patients with $\mathrm{C} 3 \mathrm{G}$, who also have a worse prognosis. Anti-factor $B$ (anti-FB) autoantibodies might be involved in the pathogenesis of APIGN, according to new findings by Véronique Frémeaux-Bacchi and colleagues.

The researchers analysed a cohort of 34 children with APIGN and noted that at diagnosis, anti-FB antibodies were detectable in $91 \%$ of patients, compared with $14 \%$ of children diagnosed with C3G $(n=28)$; C3 levels were similarly low in both groups. "We believe that screening for anti-FB antibodies in all patients diagnosed with APIGN might help discriminate patients at risk of developing $\mathrm{C} 3 \mathrm{G}$ from those with APIGN," remarks Frémeaux-Bacchi.

In patients with APIGN, anti-FB levels, which decreased over time, correlated negatively with $\mathrm{C} 3$ levels and positively with soluble C5b-9 levels. Incubation of normal human serum with IgG from patients or healthy individuals indicated that anti-FB antibodies enhance the activation of $\mathrm{C} 3$ and C5 convertases. Epitope mapping identified hotspot antibody-binding sequences in FB and revealed that antibodies specific for epitopes near the catalytic domain of FB were associated with less-severe disease. "These observations might explain the deposition of C3 that we observe in the kidneys of patients with APIGN," concludes Frémeaux-Bacchi.

Streptococcus was the most common cause of infection in patients with APIGN. "We failed to identify molecular mimicry between streptococcal antigens and FB, which suggests that a more complex mechanism underlies the pathogenesis of APIGN," explains Frémeaux-Bacchi. "We aim to identify which nephritogenic streptococcal strains are associated with anti-FB antibody formation and test their ability to activate complement in the presence of these antibodies."

Monica Wang

ORIGINAL ARTICLE Chauvet, S. et al. Anti-factor B antibodies and acute postinfectious GN in children. J. Am. Soc. Nephrol. https://doi.org/10.1681/ASN.2019080851 (2020)

\section{STONE DISEASE}

\section{Claudin 2: role in hypercalciuria and kidney stone disease}

Claudins are tight junction proteins that regulate the paracellular transport of solutes across epithelial layers. New findings demonstrate a role for the cation-selective protein claudin 2 in regulating proximal tubule (PT) calcium reabsorption and the formation of kidney stones. "Our premise, that it is defects in PT calcium handling that lead to papillary calcification and hence kidney stone disease, represents a paradigm shift in thinking about tubular calcium handling, as the scientific community has previously focused on the distal tubule as the gatekeeper for calcium excretion," explains Alan Yu. "We are not the first to postulate the importance of the PT in this process, but our study really strengthens this argument."

To assess the role of claudin 2 in calcium handling, Yu and colleagues looked at claudin 2-deficient mice. These mice were found to have hypercalciuria as a result of defective renal tubular calcium reabsorption and increased intestinal calcium absorption. The researchers also observed papillary nephrocalcinosis, with deposits resembling intratubular plugs. This resemblance prompted the researchers to examine genetic data, leading to the identification of common CLDN2 variants that were associated with decreased issue expression of claudin 2 and moderately associated with kidney stone disease risk in two large population-based studies. In one family, they identified a rare missense variant of CLDN2 that was associated with hypercalciuria and kidney stone disease.

Yu plans to further investigate the mechanisms by which claudin 2 affects calcium handling but say these insights could have consequences for treatment. "Our study suggests that interventions that increase PT calcium reabsorption, and hence reduce calcium delivery to the papilla, might be effective at reducing stone incidence," he says.

Susan J. Allison

ORIGINAL ARTICLE Curry, J. N. et al. Claudin-2 deficiency associates with hypercalciuria in mice and human kidney stone disease.J. Clin. Invest. https://doi.org/10.1172/JCl127750 (2020) 